\title{
Kinetics of molecular decomposition under irradiation of gold nanoparticles with nanosecond laser pulses-A 5-Bromouracil case study
}

Cite as: J. Chem. Phys. 152, 124712 (2020); https://doi.org/10.1063/1.5137898

Submitted: 13 November 2019 . Accepted: 21 February 2020 . Published Online: 26 March 2020

(iD) Telma S. Marques, (D) Robin Schürmann, Kenny Ebel, (D) Christian Heck, (D) Małgorzata A. Śmiałek, (D) Sam Eden, (iD) Nigel Mason, and (iD) Ilko Bald

\section{COLLECTIONS}

Paper published as part of the special topic on Emerging Directions in Plasmonics
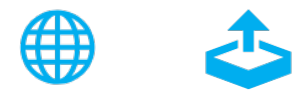

\section{ARTICLES YOU MAY BE INTERESTED IN}

Special topic on emerging directions in plasmonics

The Journal of Chemical Physics 153, 010401 (2020); https://doi.org/10.1063/5.0017914

Dark plasmon modes for efficient hot electron generation in multilayers of gold nanoparticles

The Journal of Chemical Physics 152, 064710 (2020); https://doi.org/10.1063/1.5131696

Interaction of 4-nitrothiophenol with low energy electrons: Implications for plasmon mediated reactions

The Journal of Chemical Physics 153, 104303 (2020); https://doi.org/10.1063/5.0018784

\section{Challenge us.}

What are your needs for periodic signal detection?

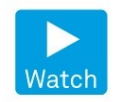

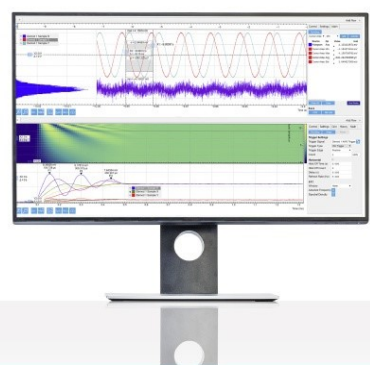

Zurich - Instruments 


\title{
Kinetics of molecular decomposition under irradiation of gold nanoparticles with nanosecond laser pulses-A 5-Bromouracil case study
}

\author{
Cite as: J. Chem. Phys. 152, 124712 (2020); doi: 10.1063/1.5137898 \\ Submitted: 13 November 2019 - Accepted: 21 February 2020 • \\ Published Online: 26 March 2020
}

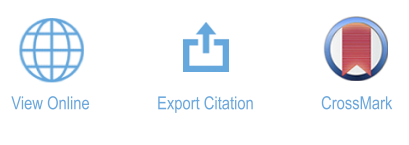

Telma S. Marques, ${ }^{1,2}$ (D) Robin Schürmann, ${ }^{3,4}$ (D) Kenny Ebel, ${ }^{3,4}$ Christian Heck, ${ }^{3}$ (D) Małgorzata A. Śmiałek, Sam Eden,' (iD Nigel Mason, ${ }^{1,6}$ (D) and Ilko Bald ${ }^{3,4, a)}$ (iD

\begin{abstract}
AFFILIATIONS
${ }^{1}$ School of Physical Sciences, The Open University, Walton Hall, MK7 6AA, Milton Keynes, United Kingdom

${ }^{2}$ CEFITEC, Departamento de Física, Faculdade de Ciências e Tecnologia, Universidade Nova de Lisboa, 2829-516 Caparica, Portugal

${ }^{3}$ Physical Chemistry, Institute of Chemistry, University of Potsdam, Karl-Liebknecht-Str. 24-25, 14476 Potsdam-Golm, Germany

${ }^{4}$ Department of Analytical Chemistry BAM, Federal Institute of Material Research and Testing, Richard-Willstätter-Str. 11, 12489 Berlin, Germany

${ }^{5}$ Department of Control and Power Engineering, Faculty of Ocean Engineering and Ship Technology, Gdansk University of Technology, Gabriela Narutowicza 11/12, 80-233 Gdansk, Poland

${ }^{6}$ School of Physical Sciences, University of Kent, Canterbury CT2 7NH, United Kingdom
\end{abstract}

Note: This paper is part of the JCP Special Topic on Emerging Directions in Plasmonics.

a) Author to whom correspondence should be addressed: bald@uni-potsdam.de

\begin{abstract}
Laser illuminated gold nanoparticles (AuNPs) efficiently absorb light and heat up the surrounding medium, leading to versatile applications ranging from plasmonic catalysis to cancer photothermal therapy. Therefore, an in-depth understanding of the thermal, optical, and electron induced reaction pathways is required. Here, the electrophilic DNA nucleobase analog 5-Bromouracil (BrU) has been used as a model compound to study its decomposition in the vicinity of AuNPs illuminated with intense ns laser pulses under various conditions. The plasmonic response of the AuNPs and the concentration of BrU and resulting photoproducts have been tracked by ultraviolet and visible (UV-Vis) spectroscopy as a function of the irradiation time. A kinetic model has been developed to determine the reaction rates of two parallel fragmentation pathways of $\mathrm{BrU}$, and their dependency on laser fluence and adsorption on the AuNP have been evaluated. In addition, the size and the electric field enhancement of the decomposed AuNPs have been determined by atomic force microscopy and finite domain time difference calculations, respectively. A minor influence of the direct photoreaction and a strong effect of the heating of the AuNPs have been revealed. However, due to the size reduction of the irradiated AuNPs, a trade-off between laser fluence and plasmonic response of the AuNPs has been observed. Hence, the decomposition of the AuNPs might be limiting the achievable temperatures under irradiation with several laser pulses. These findings need to be considered for an efficient design of catalytic plasmonic systems.
\end{abstract}

Published under license by AIP Publishing. https://doi.org/10.1063/1.5137898

\section{INTRODUCTION}

Gold nanoparticles (AuNPs) provide versatile applications in the fields of sensing, ${ }^{1,2}$ catalysis, ${ }^{3}$ and cancer therapy. ${ }^{4,5}$ Localized surface plasmons (LSPs, collective oscillations of the conduction band electrons) are responsible for the outstanding optical properties of AuNPs and can be excited by the alternating electric field of incident light. ${ }^{6}$ LSPs strongly enhance the electric field around the nanoparticle, especially when the frequency of the light matches the eigenfrequency of the LSP resonance (LSPR). LSPs can decay in a non-radiative pathway by forming electron-hole pairs, which is typically the initial step in plasmon mediated catalysis. ${ }^{3,7,8}$ Since the energy of these plasmonically generated electrons exceeds the thermal equilibrium of the electron gas, they rapidly 
distribute their energy via electron-electron scattering in the electron gas and subsequently heat up the lattice of the NPs and the surrounding medium. ${ }^{9,10}$ Under irradiation with intense ns laser pulses, the temperature of the AuNPs can be easily increased to some $1000 \mathrm{~K}$, ${ }^{11}$ which causes surface evaporation and fragmentation of the AuNPs, ${ }^{12}$ even under irradiation with a single laser shot. ${ }^{1 .}$ In this process, the morphology of the transformed AuNPs crucially depends on the irradiation parameters. ${ }^{14}$ Furthermore, a high temperature and pressure region is generated around the AuNPs if the laser intensity is sufficiently high. ${ }^{15}$ The properties of these nanobubbles are highly dependent on the size of the nanoparticles and the properties of the laser pulse. ${ }^{16,17}$ Under illumination of AuNPs with focused laser pulses, the generation of reactive secondary species such as singlet oxygen ${ }^{18}$ and low energy electrons has been obeserved. ${ }^{19}$ As high temperatures are required for the thermionic emission process, ${ }^{20}$ a threshold for the efficient generation of electrons is expected, which depends on the size of the AuNPs. Biomolecules, such as DNA ${ }^{21-23}$ or proteins, ${ }^{24-26}$ located in a nanoscopic volume around such irradiated AuNPs are efficiently decomposed under laser illumination. In that context, the adsorption of the molecules to the AuNP surface strongly influences the decomposition process. This is due to two effects: (1) the number of molecules in the high energy in the pressure region is increased and (2) the aggregation process of the AuNPs determining the size and, consequently, the plasmonic response is guided by the capping molecules. ${ }^{22,27}$ The decomposition of DNA is of particular interest for future applications in cancer photothermal therapy, where cancer cells are killed by an increase in heat mediated by incorporated laser illuminated AuNPs. ${ }^{4,28}$ The irradiation of biological tissue with intense laser pulses leads to efficient damage, as beyond the Joule heating, various nanoscopic effects around the AuNPs enhance the cellular damage. ${ }^{29}$ Even though the effects occurring in the vicinity of AuNPs illuminated with ns-laser pulses have been widely studied, their influence on biomolecules in the surrounding medium on the molecular scale is not yet fully understood. The DNA nucleobase analog 5-Bromouracil (BrU) has been proposed as a potential DNA radiosensitizer in cancer radiation therapy, ${ }^{30}$ and consequently, its reactions have been extensively studied previously. ${ }^{31,32}$ For this purpose, $\mathrm{BrU}$ will be used as a model compound to study the reactions in the vicinity of AuNPs under pulsed laser irradiation. Brominated nucleobases are known to be highly reactive with low energy electrons..$^{31,33-35}$ The attachment of an electron with a kinetic energy close to $0 \mathrm{eV}$ resonantly cleaves the carbon bromide bond of the nucleobases. ${ }^{33,35}$ This reaction has been observed recently on the surface of noble metal NPs triggered by plasmonically generated electrons. ${ }^{34}$ However, this electron induced reaction occurs close to the surface of the NPs where the highest temperatures occur, and consequently, the desorption of the reaction products without further decomposition is very unlikely.

In order to obtain an in-depth understanding of the molecular decomposition processes in the surrounding of laser illuminated AuNPs, the kinetics of different reaction pathways of $\mathrm{BrU}$ are studied as a function of irradiation related parameters within the present work. By monitoring the photoproducts of irradiated $\mathrm{BrU}$ in parallel with optical and morphological properties of the irradiated AuNPs the tunability of the plasmon catalyzed system can be evaluated.

\section{EXPERIMENTAL DETAILS}

\section{A. Chemicals}

AuNPs with $40 \mathrm{~nm}$ average diameter have been purchased from BBI solutions. BrU has been purchased from Sigma Aldrich and was dissolved in ultrapure water obtained with a Milli-Q water purification system.

\section{B. Laser irradiation}

A scheme of the experimental setup is shown in Fig. 1. Ns laser pulses have been generated using the second harmonic of a Minilite I (Continuum) Nd:YAG laser at $532 \mathrm{~nm}$ with an energy of $16 \mathrm{~mJ}$ per pulse and a pulse width of 3-5 ns. Unless stated otherwise, a repetition rate of $15 \mathrm{~Hz}$ has been used. The laser beam has been widened by using a set of two lenses from a diameter of $3 \mathrm{~mm}$ to $9 \mathrm{~mm}$. Subsequently, the beam has been guided by a dichroic mirror to a further lens $(f=5 \mathrm{~cm})$ and focused on a $3.5 \mathrm{ml}$ quartz cuvette (Hellma) slightly above the surface of the AuNP solution. The cuvette is filled with $2 \mathrm{ml}$ of solution, typically containing $45 \mathrm{pM}$ AuNPs and 40 $\mu \mathrm{M} \mathrm{BrU}$, and placed on a stirring plate to stir the solution during the irradiation. Using a mechanical stage, the distance of the laser focus to the surface of the AuNP solution has been varied to adjust the spot size and, in consequence, the laser fluence of the divergent

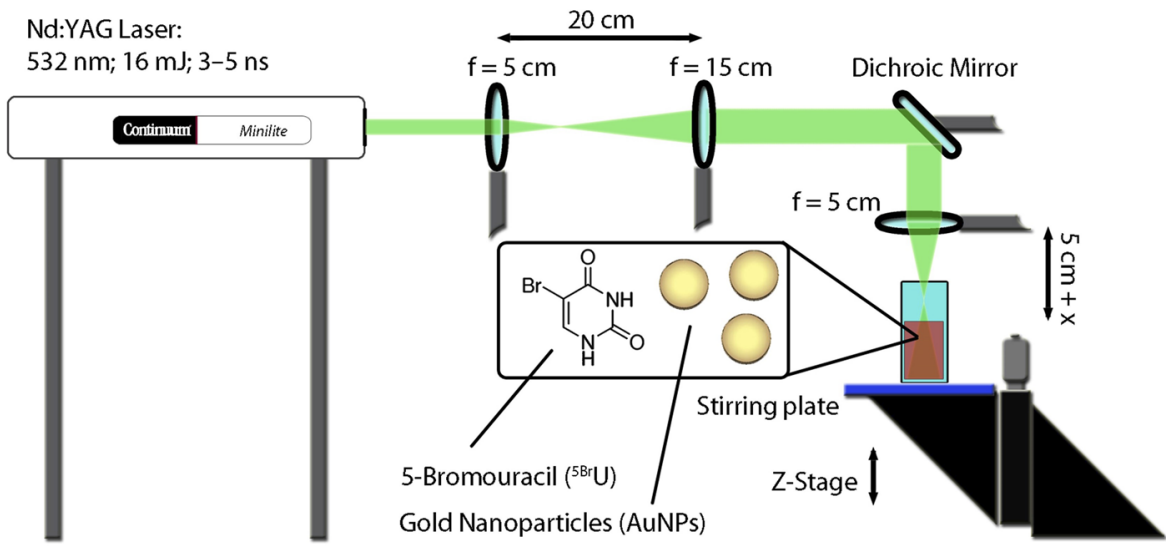

FIG. 1. Schematic representation of the experimental setup. 
beam on the surface. The laser fluences given below refer to the maximum laser fluence at the surface, without considering the widening of the beam inside the cuvette. The size of the focused laser beam has been determined using an optical microscope and a blackened photographic paper irradiated with a single laser shot.

\section{Analytical methods}

Ultraviolet and visible (UV-Vis) extinction spectra were recorded with a Jasco 650 spectrophotometer. Dried AuNPs have been imaged with an Agilent 5500 atomic force microscope (AFM) using a Tap 150 cantilever in the tapping mode. For the sample preparation, a $2 \mu \mathrm{l}$ droplet of the irradiated AuNP solution has been dried on a freshly cleaved mica substrate. The size distribution of the AuNPs on the substrate has been determined from the height of the AuNPs in the AFM images by using the software Gwyddion 2.48.

\section{FDTD calculations}

Finite domain time difference (FDTD) calculations of the electric field enhancement of AuNPs in an aqueous medium have been carried out with the software Lumerical FDTD Solutions 8.6.3, using a mesh size of $0.1 \mathrm{~nm}$ in the plotted areas. The excitation wavelength was set to $532 \mathrm{~nm}$.

\section{RESULTS AND DISCUSSIONS}

Using the experimental setup presented in Fig. 1, a mixture of AuNPs and BrU has been irradiated with ns laser pulses. UV-Vis spectra have been recorded after specific illumination times in order to determine the LSPR of the AuNPs and the $\pi-\pi^{*}$ resonance of $\mathrm{BrU}$. In Fig. 2(a), a typical dataset is presented showing that the LSPR, which is initially located at $528 \mathrm{~nm}$, is decreased and blue shifted with ongoing irradiation. This change in the LSPR is caused by the decomposition of the AuNPs into smaller fragments. Already after 5 min of irradiation, only small changes in the LSPR are observable, which indicates only slight changes in the size distribution, and thus, approximately constant reaction conditions for the molecular decomposition can be assumed. Moreover, also the intensity of the $\pi-\pi^{*}$ transition of $\mathrm{BrU}$, located at $277 \mathrm{~nm},{ }^{36}$ is reduced and shifted to a lower wavelength during the irradiation. The decrease in the $\pi-\pi^{*}$ resonance is attributed to a cleavage of the aromatic ring structure, whereas the shift of the resonance maximum indicates a chemical modification of the $\mathrm{BrU}$, most likely the cleavage of the $\mathrm{C}-\mathrm{Br}$ bond leaving the residual molecule intact.

In order to further analyze the $\pi-\pi^{*}$ transition, all additional contributions of the solution to the extinction in this wavelength regime need to be determined to correct the background of the BrU spectra [see Fig. 2(b)]. Therefore, AuNPs have been irradiated in the absence of BrU under the same experimental conditions, as the absorption of AuNPs in the UV caused by interband transitions depends significantly on the particle size (see Fig. SI2).

The spectra of the irradiated AuNP solution have been subtracted from the spectra of the irradiated AuNP/BrU solution for each irradiation time. In addition, the absorption band located below $210 \mathrm{~nm}$ has been fitted with a Gaussian peak and subtracted from the AuNP/BrU spectra, since there are slight contributions of these signals to the $\pi-\pi^{*}$ peak. To determine the contributions of the $\pi-\pi^{*}$ transitions of $\mathrm{BrU}$ and $\mathrm{U}$, the background corrected AuNP/BrU spectra of the $\pi-\pi^{*}$ transition, shown in Fig. 2(c), have been fitted with two Gaussian peaks centered at $277 \mathrm{~nm}$ and $258 \mathrm{~nm},{ }^{37}$ respectively. In this way, the concentration of $\mathrm{BrU}[\mathrm{BrU}]$ and of $U[U]$ can be monitored as a function of the irradiation time.

In order to explain the shift and the decrease of the $\pi-\pi^{*}$ signal, two reaction pathways are assumed: the fragmentation of the molecular ring leading to a decrease in the $\pi-\pi^{*}$ resonance,

$$
\mathrm{BrU} \stackrel{k 1}{\rightarrow} \text { small fragments, }
$$

and the cleavage of the $\mathrm{C}-\mathrm{Br}$ bond resulting in the formation of Uracil (U),

$$
\mathrm{BrU} \stackrel{k 2}{\rightarrow} \mathrm{U}
$$

where $k_{1}$ and $k_{2}$ denote the reaction rates for the fragmentation of the ring and the cleavage of the $\mathrm{C}-\mathrm{Br}$ bond, respectively. In addition, a third reaction with a reaction rate $k_{3}$ needs to be considered, since the generated $\mathrm{U}$ will also be decomposed under laser irradiation in the presence of AuNPs into smaller fragments,

$$
\mathrm{U} \stackrel{k 3}{\rightarrow} \text { small fragments. }
$$
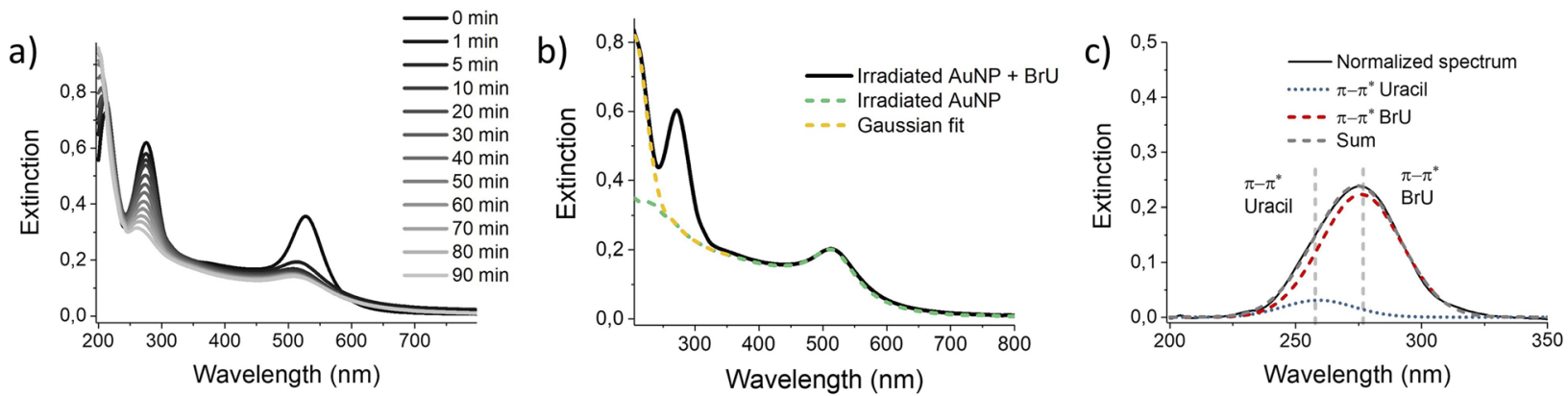

FIG. 2. (a) UV-Vis spectra of the AuNP/BrU solution irradiated with focused $532 \mathrm{~nm}$ ns laser pulses with a repetition rate of $15 \mathrm{~Hz}$ and a maximum laser fluence of $3.4 \times 10^{12} \mathrm{~W} / \mathrm{m}^{2}$. (b) UV-Vis spectra of the irradiated AuNP/BrU solution (black), a spectrum of illuminated AuNPs under the same conditions (green, dashed), and a Gaussian fit of the peak centered below $210 \mathrm{~nm}$ (yellow, dashed). (c) $\pi-\pi^{*}$ signal (black) corrected by the contributions marked in (b). The Gaussian fit of the contributions of $\mathrm{U}$ (blue) and $\mathrm{BrU}$ (red) and their sum (gray) are plotted with dotted lines. 
Based on Eqs. (1) and (2), the decomposition of $\mathrm{BrU}$ follows a (pseudo-)first order reaction that can be described by the following equation:

$$
\frac{d[\mathrm{BrU}]}{d t}=-k_{1}[\mathrm{BrU}]-k_{2}[\mathrm{BrU}] .
$$

According to Eqs. (2) and (3), the generation and decomposition of $\mathrm{U}$ can be described by

$$
\frac{d[\mathrm{U}]}{d t}=-k_{3}[\mathrm{U}]+k_{2}[\mathrm{BrU}] .
$$

From Eq. (4), we obtain for the concentration of BrU, [BrU], after an irradiation time $t$,

$$
[\mathrm{BrU}]=[\mathrm{BrU}]_{0} e^{-\left(k_{1}+k_{2}\right) t},
$$

where $[\mathrm{BrU}]_{0}$ is the initial concentration of $\mathrm{BrU}$ before the irradiation. Since there has been initially no $U$ in the solution, we set $\left[\mathrm{U}_{0}\right]=0$ and obtain $[\mathrm{U}]$ (see the supplementary material for details),

$$
[\mathrm{U}]=\frac{k_{2}[\mathrm{BrU}]_{0}}{k_{3}-k_{1}-k_{2}}\left(e^{-\left(k_{1}+k_{2}\right) t}-e^{-k_{3} t}\right) .
$$

Consequently, the ratio of $[\mathrm{U}]$ and $[\mathrm{BrU}]$ can be determined using Eqs. (6) and (7),

$$
\frac{[\mathrm{U}]}{[\mathrm{BrU}]}=\frac{k_{2}}{k_{3}-k_{1}-k_{2}}\left(1-e^{-\left(k_{3}-k_{1}-k_{2}\right) t}\right)
$$

By using the Taylor expansion $e^{x} \approx 1+x$, we can simplify the expression for short illumination times $t$ to

$$
\frac{[\mathrm{U}]}{[\mathrm{BrU}]} \approx k_{2} t .
$$

With this equation, the reaction rate $k_{2}$ can be determined from the ratio of the concentrations $[\mathrm{BrU}]$ and $[\mathrm{U}]$, which can be determined from the intensity of the $\pi-\pi^{*}$ resonances at $258 \mathrm{~nm}$ and $277 \mathrm{~nm}$ in the UV-Vis spectra as a function of the irradiation time $t$ [see Fig. 3(a)]. Error bars have been determined from the background subtraction of the AuNP signal in the absence of $\mathrm{BrU}$, and the fits are presented in Figs. 2(b) and 2(c). Due to the strong changes in the AuNP size after the influence of first laser pulses (see the text below and Fig. 6) on the reaction conditions, the data points after 0 min and 1 min irradiation times have not been taken into account in the analysis. The determined ratio of [BrU] and [U] typically follows the expected linear trend after the particle size remains constant.
In Fig. 3(b), [BrU] is plotted as a function of $t$ and fitted with an exponential decay; hence, the sum of the reaction rates $k_{1}$ and $k_{2}$ can be determined from the fit by using Eq. (6). However, for short and very long irradiations times, the exponential correlation is only valid in a first approximation due to the reduced signal to background ratio of the $\pi-\pi^{*}$ resonance for long illumination times. Therefore, based on the experimental data, it cannot be finally excluded that the reaction might also follow a zeroth or some more complex reaction order.

The reaction rates $k_{1}$ and $k_{2}$ have been determined at a fixed laser fluence for different repetition rates of the laser. The error bars of the reaction rates $k_{1}$ and $k_{2}$ originate from the fit presented in Fig. 3 (see Fig. 4). The reaction rate $k_{1}$ decreases significantly with higher laser repetition rates. At higher laser repetition rates, the time between two subsequent pulses is shorter. In consequence, there is less time following a laser pulse in which the BrU can adsorb on the cleaned surface before the AuNP is illuminated again. Therefore, the coverage of $\mathrm{BrU}$ on the AuNP surface during the pulse is lower at higher laser repetition rates as the adsorption time for $\mathrm{BrU}$ is shortened. Lower concentrations of BrU on the AuNPs, i.e., in the areas of the highest temperatures, result in decreasing reaction rates for $k_{1}$. This trend is in accordance with the results published previously. ${ }^{22}$ Nevertheless, for $k_{2}$, no dependency on the repetition rate has been observed. $k_{2}$ represents the $\mathrm{C}-\mathrm{Br}$ bond cleavage leaving the $\mathrm{U}$ ring intact. The $\mathrm{C}-\mathrm{Br}$ bond of $\mathrm{BrU}$ is a predetermined breaking point of the molecule and can be efficiently cleaved by the dissociative attachment of low energy electrons, and also it is the first bond to break at elevated temperatures. ${ }^{38}$ However, both processes might occur as well in the vicinity of the AuNP surface and do not require adsorption of the molecules. Furthermore, the conditions directly on the AuNP surface are extreme in terms of temperature and pressure. Hence, it is unlikely that, in adsorbed BrU molecules, only the $\mathrm{C}-\mathrm{Br}$ bond will be cleaved under laser irradiation prior desorption leaving the $U$ ring intact.

Moreover, the reaction rates have been determined as a function of the maximum laser fluence [see Fig. 5(a)]. For this purpose, the distance from the surface of the AuNP/BrU solution to the focus of the laser beam was varied using a mechanical stage. Due to this setup, the photon fluence can be varied while keeping the power of the laser pulses constant. Even though the laser pulses have a Gaussian shape and the divergent laser beam widens during the passage through the AuNP/BrU solution leading to a spatially inhomogeneously distributed fluence, the maximum laser fluence is proportional to the average fluence in the solution. The reaction
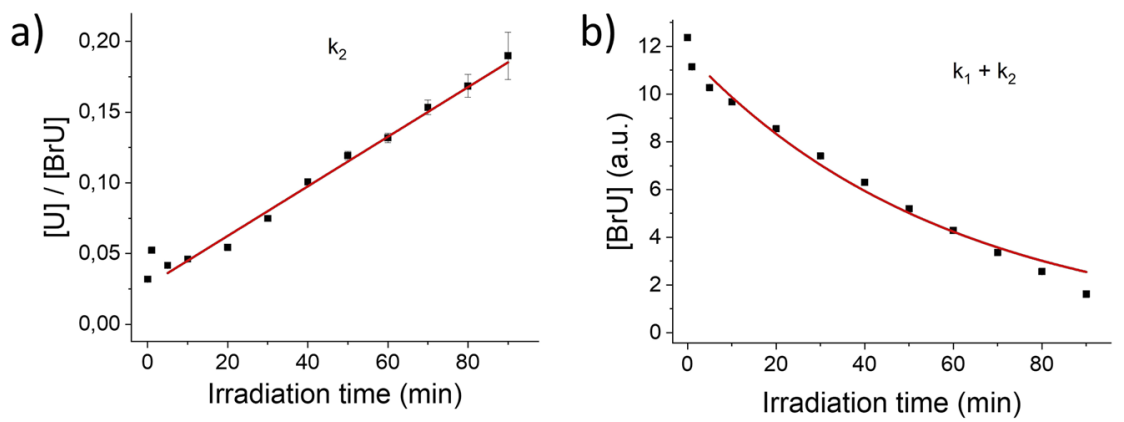

FIG. 3. (a) Ratio of the concentrations $[\mathrm{U}]$ and $[\mathrm{BrU}]$ plotted against the irradiation time $t$ fit linearly to determine $k_{2}$ from the slope. (b) Concentration [BrU] plotted as a function of the irradiation time $t$ and fit with an exponential decay curve to determine $k_{1}$ and $k_{2}$ from the decay constant. 


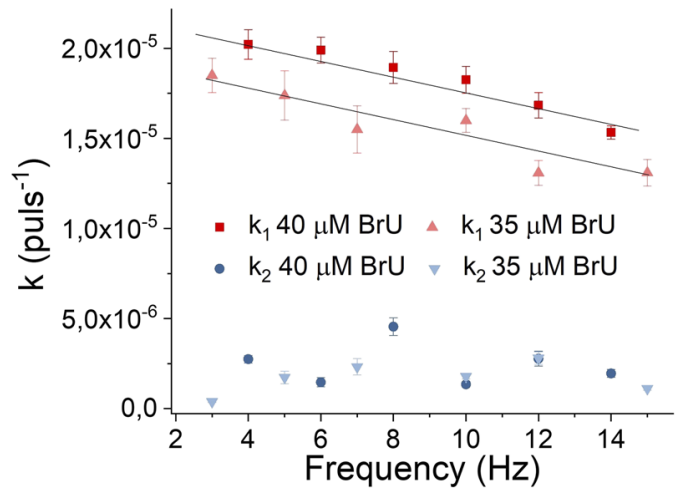

FIG. 4. Reaction rates $k_{1}$ (red) and $k_{2}$ (blue) of $\mathrm{BrU}$ as a function of the laser repetition rate for two different initial concentrations of $\mathrm{BrU}$ (dark and light, respectively). Black lines are plotted as guides for the eye.

rates $k_{1}$ and $k_{2}$ show the same behavior as a function of the laser fluence, whereas $k_{1}$ is typically almost one order of magnitude higher than $k_{2}$. Up to a laser fluence of around $10^{13} \mathrm{~W} / \mathrm{m}^{2}$, the reaction rates increase with the laser fluence; however, for higher fluences $\left(>10^{13} \mathrm{~W} / \mathrm{m}^{2}\right)$, the reaction rates decrease. A threshold, where $k_{2}$ is significantly increased with respect to $k_{1}$ due to an enhanced generation of thermionic electrons as predicted previously by Pyatenko et al., has not been observed. ${ }^{20}$ In the predictions of the threshold, nanoparticles of constant size have been assumed; however, the size of the generated nanoparticle fragments significantly depends on the laser fluences. The size of the nanoparticles strongly influences the absorption of the AuNPs at $532 \mathrm{~nm}$ due to the LSPR. In Fig. 5(b), the absorbance at a wavelength of $532 \mathrm{~nm}$ has been plotted against the laser power, showing a decrease in the absorbance with an increase in the laser fluence reaching a minimum at $\sim 10^{13} \mathrm{~W} / \mathrm{m}^{2}$ as well. In general, small AuNPs exhibit LSPRs with lower intensities, which are centered at a comparably lower wavelength. The decrease in the LSPRs as a function of the laser power indicates a stronger fragmentation of the AuNPs at higher laser powers. Nevertheless, the resulting decreased absorption at $532 \mathrm{~nm}$ results in a lower energy absorption by the AuNP solution, limiting the heating of the AuNPs and leading to stagnation or even reduction of the reaction rates at high fluences. At high laser fluences, especially for the irradiation of larger AuNPs, the ignition of plasmas has been observed. ${ }^{39}$ Since the plasmas occur statistically, their role in the decomposition of the AuNPs and the molecules could not be evaluated.

Although the LSPR absorption decreases with higher laser fluence, the reaction rates increase up to a maximum fluence of $10^{13} \mathrm{~W} / \mathrm{m}^{2}$. This might be explained by an increased surface area of the smaller AuNPs, since the number of gold atoms in the solution remains constant under the irradiation. It is also possible that higher temperature around the AuNPs might be responsible for the higher reaction rates. In order to determine the surface area $A_{S}$ of the AuNPs, the size distribution of the AuNPs after an irradiation for $20 \mathrm{~min}$ has been determined by AFM for six different laser fluences. Figures 6(a) and 6(b) show typical AFM images of the AuNPs after irradiation. The diameter of the AuNPs has been determined from the height of the AuNPs, and the normalized size distributions of the AuNPs are shown in Fig. 6(c). 500-3000 AuNPs have been analyzed at each of the laser fluences studied. In all cases, the size of the AuNPs after the irradiation was reduced from $40 \mathrm{~nm}$ to below $10 \mathrm{~nm}$. With the knowledge of the normalized size distribution of the AuNPs, the overall surface area of the AuNPs in the solution can be calculated using the following equation:

$$
A_{S}=\frac{V_{\text {gold }}}{\sum P(r) \cdot V(r)} \cdot 4 \pi \cdot \sum P(r) \cdot r^{2},
$$

where $V_{\text {gold }}$ is the total volume of the AuNPs in the solution, $r$ is the radius of the AuNPs, $P(r)$ is the percentage of AuNPs with a radius $r$ in the solution determined from the histograms shown in Fig. 6(c) and Fig. SI3, and $V(r)$ is the volume of an AuNP with a radius $r$. In Fig. 6(d), the surface area is plotted as a function of the laser fluence revealing an increase in the surface area with the laser fluence. The error bars originate from the statistical error of the AuNP counting (see the supplementary material for details).

It should be noted that at higher laser fluences, a smaller fraction of the solution is illuminated. In consequence, the illuminated surface area $A_{\text {ill }}$ of the AuNPs needs to be determined to evaluate the effect of the surface area on the reaction rates. For this purpose, the illuminated volume $V_{\text {ill }}$ for certain laser fluences has been calculated using basic geometrical optics assuming a simplified model of
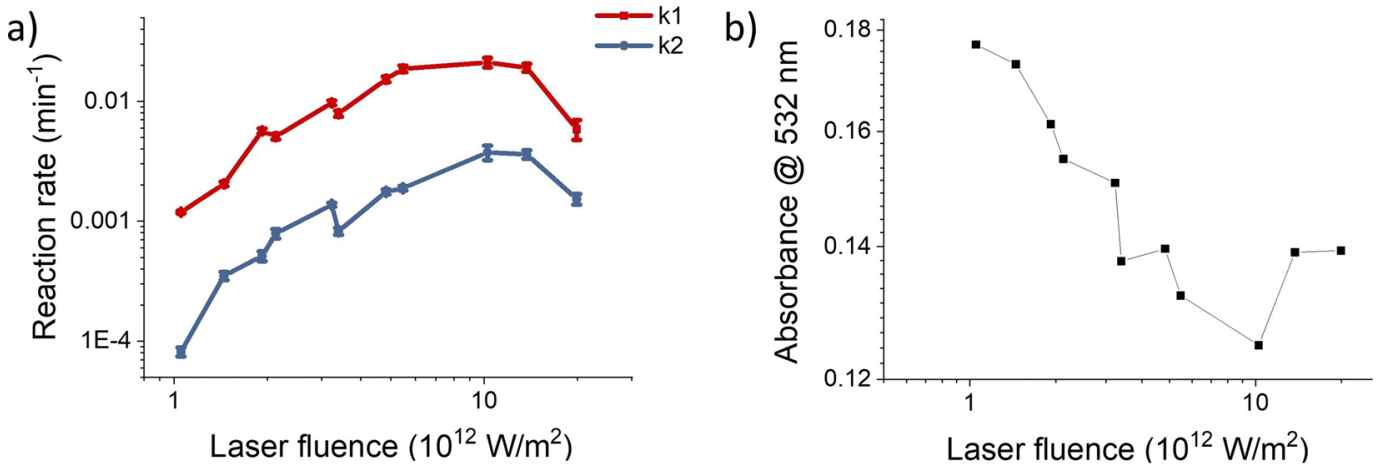

FIG. 5. (a) Reaction rates $k_{1}$ and $k_{2}$ plotted as a function of the laser fluence. (b) Absorbance of the AuNP/BrU solution at $532 \mathrm{~nm}$ after 20 min irradiation plotted against the laser fluence. 
a)

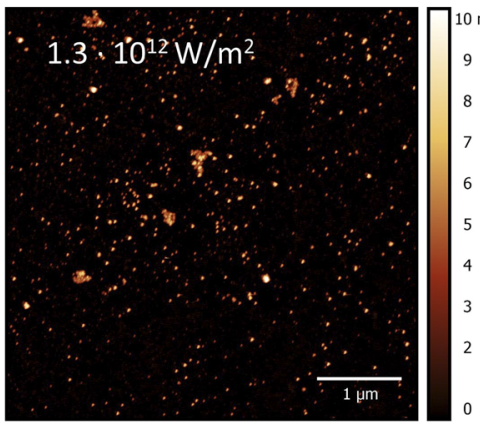

d)

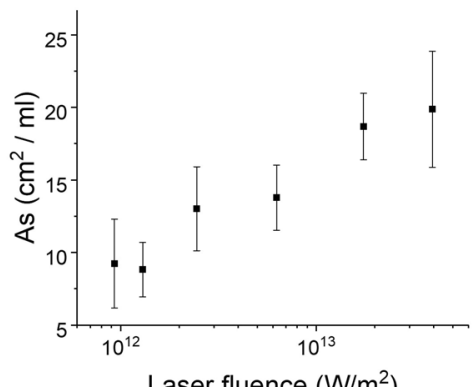

b)

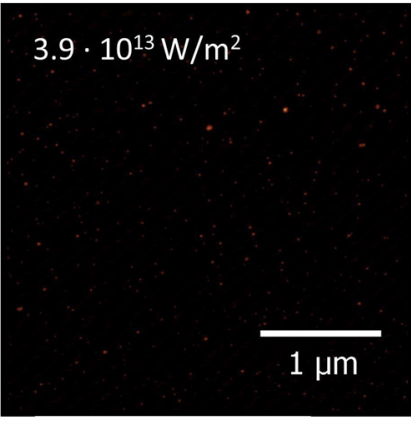

e)

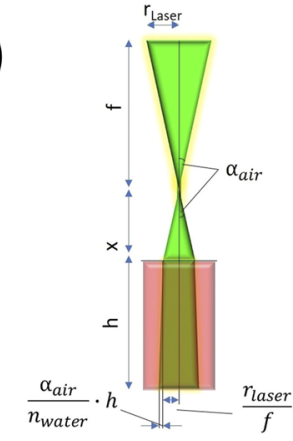

c)

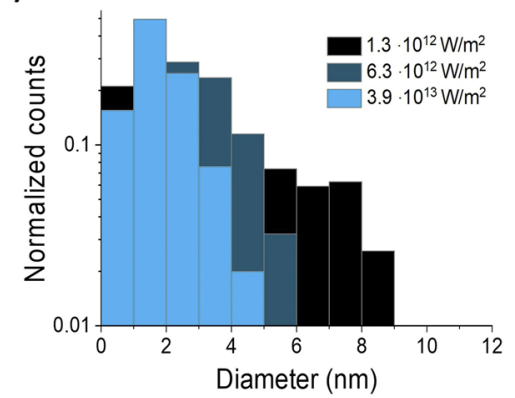

f)

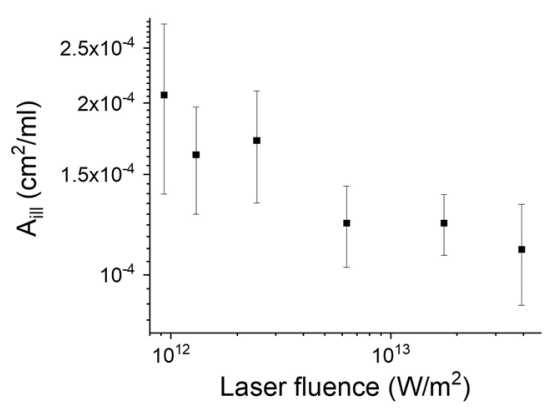

FIG. 6. AFM image of AuNPs dried on a mica substrate illuminated for 20 min with a maximum laser fluence of (a) $1.310^{12}$ W/m² and (b) $3.910^{13}$ W/m² , (c) Normalized size distribution of AuNPs irradiated using different laser fluences. All histograms are presented individually in Fig. SI3. (d) Surface area of the irradiated AuNPs determined using Eq. (12) as a function of the laser fluence. (e) Sketch of the laser beam propagation after passing the focusing lens according to geometrical optics. (f) Illuminated surface area during one laser pulse as a function of the laser fluence after irradiation for $20 \mathrm{~min}$.

the laser beam path. The beam path in the solution has a truncated cone shape. A sketch of the beam propagation after passing the final focusing lens is presented in Fig. 6(e). The illuminated volume can be calculated by

$$
\begin{aligned}
V_{\text {ill }}= & \frac{\pi \cdot h}{3}\left[\left(\frac{x}{f} \cdot r_{\text {Laser }}\right)^{2}+\left(\frac{x}{f} \cdot r_{\text {Laser }}\right)\right. \\
& \left.\times\left(\frac{r_{\text {Laser }}}{f \cdot n_{\text {water }}} \cdot h+\frac{x}{f} \cdot r_{\text {Laser }}\right)+\left(\frac{r_{\text {Laser }}}{f \cdot n_{\text {water }}} \cdot h+\frac{x}{f} \cdot r_{\text {Laser }}\right)^{2}\right] .
\end{aligned}
$$

From the ratio of $V_{\text {ill }}$ to the total volume of the solution $V_{\text {total }}$, the illuminated area $A_{\text {ill }}$ can be calculated by using Eq. (11),

$$
A_{\text {ill }}=\frac{V_{\text {ill }}}{V_{\text {total }}} \cdot A_{S}
$$

In Fig. 6(f), $A_{i l l}$ is plotted as a function of the laser fluence, revealing a decrease in $A_{i l l}$ with the fluence, although the relative error of the calculation is large. Hence, the increased reaction rates at higher laser fluences might not be solely explained by an increased surface area of the illuminated AuNPs.

Therefore, the absorbed heat $Q_{a b s}$ of an individual AuNP during a laser pulse has been calculated from the absorption cross section $\sigma_{a b s}$ of the irradiated AuNPs and the laser fluence I,

$$
Q_{a b s}=\sigma_{a b s} \cdot I \text {. }
$$

For small nanoparticles, mainly the absorption is contributing to the extinction and the scattering can be neglected. Thus, the UV-Vis data presented in Fig. 5(b) have been used to estimate the absorption cross section $\sigma_{a b s}$ of the irradiated AuNP solution by using the Lambert-Beer law,

$$
A b s=l \cdot \sigma_{a b s} \cdot N_{\mathrm{AuNP}} .
$$

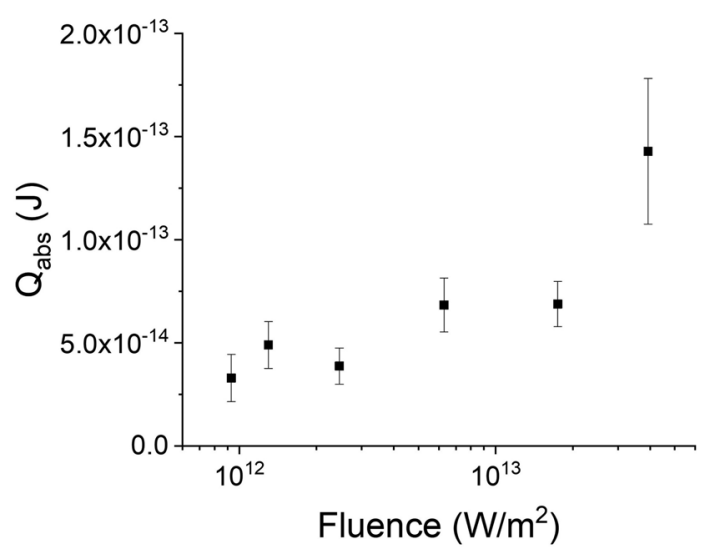

FIG. 7. Heat absorbed by a single AuNP in one $16 \mathrm{~mJ}$ laser pulse plotted as a function of the laser fluence. 

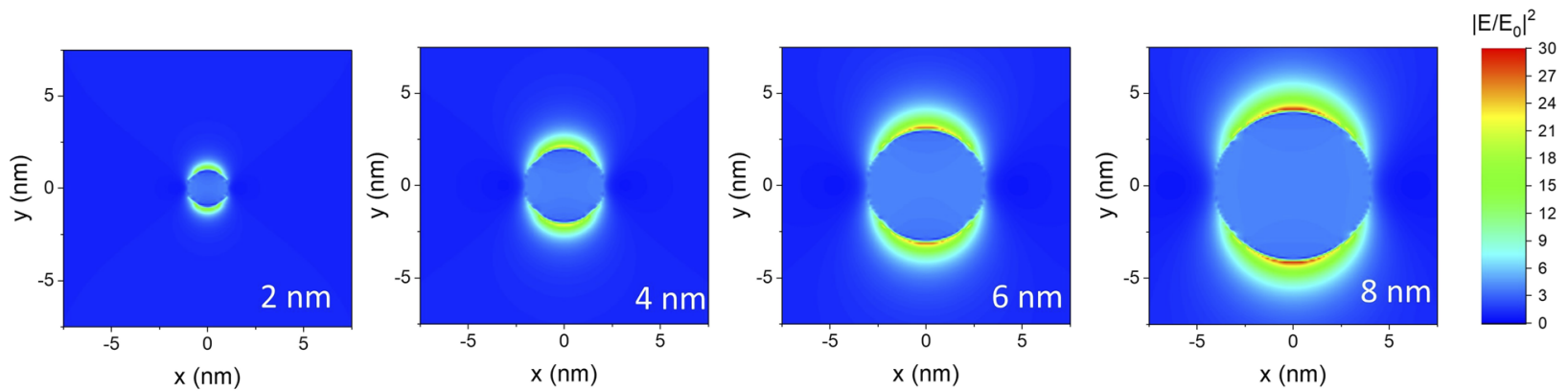

FIG. 8. FDTD-calculation square of the electric field enhancement $\left|E / E_{0}\right|^{2}$ of AuNPs with diameters of $2 \mathrm{~nm}, 4 \mathrm{~nm}, 6 \mathrm{~nm}$, and $8 \mathrm{~nm}$, respectively, in an aqueous medium at a wavelength of $532 \mathrm{~nm}$.

The number of AuNPs per unit volume $N_{\text {AuNP }}$ of the irradiated AuNP solution has been determined by

$$
N_{\mathrm{AuNP}}=\frac{V_{\mathrm{AuNP} 40 \mathrm{~nm}}}{\sum P(r) \cdot V(r)} \cdot N_{\mathrm{AuNP} 40 \mathrm{~nm}}
$$

where $V_{\text {AuNP } 40 \mathrm{~nm}}$ is the volume of a AuNP with a diameter of $40 \mathrm{~nm}$ and $N_{\text {AuNP } 40 \mathrm{~nm}}$ is the initial number of $40 \mathrm{~nm}$ AuNPs per unit volume in the solution prior to irradiation.

In Fig. 7, $Q_{\mathrm{abs}}$ is plotted as a function of the laser fluence revealing an increase in $Q_{a b s}$ with the laser fluence leading to a higher temperature of the individual AuNPs. Hence, the increase in the reaction rates with the laser fluence might be caused by an increased temperature around the AuNPs and possibly by an increased generation of reactive species, such as low energy electrons, even though the total absorption of light in the solution [see Fig. 5(b)] and the illuminated surface area [see Fig. 6(f)] are smaller. However, the time between two laser pulses illuminating the same AuNP is longer for a smaller illuminated area, and furthermore, the temperature gradient leads to migration of molecules toward the AuNP surface; ${ }^{40}$ thus, the effect of adsorption on the decomposition rate cannot be neglected in this context.

In order to evaluate the possible impact of multi-photon processes, the enhancement of the electrical field $\left|E / E_{0}\right|^{2}$ around the AuNPs has been determined by FDTD simulations. The simulations have been performed for spherical AuNPs with a diameter between $2 \mathrm{~nm}$ and $8 \mathrm{~nm}$, which are typically generated under the present experimental conditions. In Fig. 8, $\left|E / E_{0}\right|^{2}$ is plotted in the $\mathrm{x}-\mathrm{y}$ plane crossing the center of the particle. For AuNPs with a diameter of $2 \mathrm{~nm}$, the intensity enhancement is comparably small, not exceeding a factor of $\sim 15$ at the spots with the highest enhancement. Furthermore, the spots with a high enhancement are highly localized close to the particle surface. At a distance of around $1 \mathrm{~nm}$ from the surface, a significant enhancement of $\left|E / E_{0}\right|^{2}$ is no longer observable. With an increase in the diameter of the AuNPs, the intensity enhancement in the vicinity of the particles is increased. However, even for AuNPs with a diameter of $8 \mathrm{~nm}$, the maximum intensity enhancement does not exceed a factor of 30. Irradiation of a BrU solution for several hours at high laser fluences in the absence of AuNPs did not lead to a change in the $\pi-\pi^{*}$ resonance (see Fig. SI1). For uracil and thymine, a threshold multiphoton excitation leading to the fragmentation of the molecules has been observed at energies involving at least three $532 \mathrm{~nm}(2.33 \mathrm{eV})$ photons. ${ }^{41,42}$ In consequence, it is very unlikely to observe a significant contribution of multiphoton effects due to the laser irradiation in the present experiments, as the volumes with a comparably high enhancement are very localized and correlate with the volumes where the highest temperatures will also occur; thus, a thermal decay of possible photoproducts is likely.

\section{CONCLUSION}

In summary, the kinetics of two decomposition pathways of BrU adsorbed on AuNPs induced by pulsed laser illumination have been tracked by UV-Vis spectroscopy and reaction rates have been determined using a kinetic model. The decomposition rates for the fragmentation of the $\mathrm{BrU}$ ring structure depend on the fluence, the repetition rate of the laser, and the starting concentration of $\mathrm{BrU}$. At higher laser fluences, the AuNPs are decomposed and the surface area is increased, also leading to higher temperatures. However, on the other hand, the irradiated volume is decreased and the plasmonic response is significantly lowered. Hence, these opposing effects lead to a trade-off limiting the decomposition rates. The cleavage of the $\mathrm{C}-\mathrm{Br}$ bond leaving the residual molecule intact is most likely independent of the adsorption of the molecules on the AuNPs. This process is probably electron or thermally induced, and multiphoton excitation processes are very unlikely. As the irradiation parameters are interlinked with the optical and thermal properties of the generated AuNP substrates, the tunability of the reaction kinetics of the system is only limited.

\section{SUPPLEMENTARY MATERIAL}

See the supplementary material for the complete derivation of the reaction kinetics, irradiation of $\mathrm{BrU}$ in the absence of AuNPs, and error calculation.

\section{AUTHOR'S CONTRIBUTIONS}

T.S.M. and R.S. contributed equally. 


\section{ACKNOWLEDGMENTS}

The authors acknowledge the financial support from the Deutsche Forschungsgemeinschaft (Project No. 230710387), Fundação para a Ciência e a Tecnologia (FCT-MCTES), Radiation Biology and Biophysics Doctoral Training Programme (RaBBiT, PD/00193/2012), Applied Molecular Biosciences Unit - UCIBIO (UIDB/04378/2020) and CEFITEC Unit (UIDB/00068/2020), and scholarship grant number SFRH/BD/106032/2015 to Telma S. Marques.

\section{REFERENCES}

1'J. R. Mejía-Salazar and O. N. Oliveira, Chem. Rev. 118, 10617 (2018).

${ }^{2}$ K. A. Willets and R. P. van Duyne, Annu. Rev. Phys. Chem. 58, 267 (2007).

${ }^{3}$ Y. Zhang, S. He, W. Guo, Y. Hu, J. Huang, J. R. Mulcahy, and W. D. Wei, Chem. Rev. 118, 2927 (2018).

${ }^{4}$ N. S. Abadeer and C. J. Murphy, J. Phys. Chem. C 120, 4691 (2016).

${ }^{5}$ K. Haume, S. Rosa, S. Grellet, M. A. Śmiałek, K. T. Butterworth, A. V. Solov'yov, K. M. Prise, J. Golding, and N. J. Mason, Cancer Nanotechnol. 7, 8 (2016).

${ }^{6}$ D. Lin, R. He, S. Li, Y. Xu, J. Wang, G. Wei, M. Ji, and X. Yang, ACS Chem. Neurosci. 7, 1728 (2016).

${ }^{7}$ E. Cortés, Adv. Opt. Mater. 5, 1700191 (2017).

${ }^{8}$ R. Schürmann, K. Ebel, C. Nicolas, A. R. Milosavljević, and I. Bald, J. Phys. Chem. Lett. 10, 3153 (2019).

${ }^{9}$ S. Hashimoto, D. Werner, and T. Uwada, J. Photochem. Photobiol. C: Photochem. Rev. 13, 28 (2012).

${ }^{10}$ Z. Qin and J. C. Bischof, Chem. Soc. Rev. 41, 1191 (2012).

${ }^{11}$ M. Strasser, K. Setoura, U. Langbein, and S. Hashimoto, J. Phys. Chem. C 118, 25748 (2014).

${ }^{12}$ G. González-Rubio, A. Guerrero-Martínez, and L. M. Liz-Marzán, Acc. Chem. Res. 49, 678 (2016).

${ }^{13}$ A. R. Ziefuß, S. Reichenberger, C. Rehbock, I. Chakraborty, M. Gharib, W. J. Parak, and S. Barcikowski, J. Phys. Chem. C 122, 22125 (2018).

${ }^{14}$ D. Zhang, B. Gökce, and S. Barcikowski, Chem. Rev. 117, 3990 (2017).

${ }^{15}$ S. Hashimoto, T. Katayama, K. Setoura, M. Strasser, T. Uwada, and H. Miyasaka, Phys. Chem. Chem. Phys. 18, 4994 (2016).

${ }^{16}$ K. Metwally, S. Mensah, and G. Baffou, J. Phys. Chem. C 119, 28586 (2015).

${ }^{17}$ S. Wang, L. Fu, Y. Zhang, J. Wang, and Z. Zhang, J. Phys. Chem. C 122, 24421 (2018).
${ }^{18}$ S. J. Chadwick, D. Salah, P. M. Livesey, M. Brust, and M. Volk, J. Phys. Chem. C 120, 10647 (2016).

${ }^{19}$ K. Yamada, K. Miyajima, and F. Mafuné, J. Phys. Chem. C 111, 11246 (2007).

${ }^{20}$ A. Pyatenko, M. Yamaguchi, and M. Suzuki, J. Phys. Chem. C 113, 9078 (2009).

${ }^{21}$ R. Schürmann and I. Bald, J. Phys. Chem. C 120, 3001 (2016).

${ }^{22}$ R. Schürmann and I. Bald, Phys. Chem. Chem. Phys. 19, 10796 (2017).

${ }^{23}$ Y. Takeda, T. Kondow, and F. Mafuné, Phys. Chem. Chem. Phys. 13, 586 (2011).

${ }^{24}$ F. Sauvage, J. Schymkowitz, F. Rousseau, B. Z. Schmidt, K. Remaut, K. Braeckmans, and S. C. de Smedt, Nano Today (in press).

${ }^{25}$ Y. Takeda, T. Kondow, and F. Mafuné, J. Phys. Chem. B 110, 2393 (2006).

${ }^{26}$ Y. Takeda, F. Mafuné, and T. Kondow, J. Phys. Chem. C 113, 5027 (2009).

${ }^{27}$ T. Tsuji, S. Sakaki, H. Fujiwara, H. Kikuchi, M. Tsuji, Y. Ishikawa, and N. Koshizaki, J. Phys. Chem. C 122, 21659 (2018).

${ }^{28}$ M. Aioub and M. A. El-Sayed, J. Am. Chem. Soc. 138, 1258 (2016).

${ }^{29}$ E. Boulais, R. Lachaine, A. Hatef, and M. Meunier, J. Photochem. Photobiol. C: Photochem. Rev. 17, 26 (2013).

${ }^{30}$ M. D. Prados, W. Seiferheld, H. M. Sandler, J. C. Buckner, T. Phillips, C. Schultz, R. Urtasun, R. Davis, P. Gutin, T. L. Cascino, H. S. Greenberg, and W. J. Curran, Int. J. Radiat. Oncol., Biol., Phys. 58, 1147 (2004).

${ }^{31}$ J. Rak, L. Chomicz, J. Wiczk, K. Westphal, M. Zdrowowicz, P. Wityk, M. Żyndul, S. Makurat, and Ł. Golon, J. Phys. Chem. B 119, 8227 (2015).

${ }^{32}$ M. Zdrowowicz, B. Michalska, A. Zylicz-Stachula, and J. Rak, J. Phys. Chem. B 118, 5009 (2014).

${ }^{33}$ H. Abdoul-Carime, M. A. Huels, F. Brüning, E. Illenberger, and L. Sanche, J. Chem. Phys. 113, 2517 (2000).

${ }^{34}$ R. Schürmann and I. Bald, Nanoscale 9, 1951 (2017).

${ }^{35}$ R. Schürmann, K. Tanzer, I. Dąbkowska, S. Denifl, and I. Bald, J. Phys. Chem. B 121, 5730 (2017).

${ }^{36}$ D. B. Dunn and J. D. Smith, Biochem. J. 67, 494 (1957).

${ }^{37}$ G. D. Fasman, CRC Handbook of Biochemistry and Molecular Biology (CRC Press, 2019).

${ }^{38}$ J. C. Tully, Science 312, 1004 (2006).

${ }^{39}$ A. A. Serkov, P. G. Kuzmin, I. I. Rakov, and G. A. Shafeev, Quantum Electron. 46, 713 (2016).

${ }^{40}$ M. Enders, S. Mukai, T. Uwada, and S. Hashimoto, J. Phys. Chem. C 120, 6723 (2016).

${ }^{41}$ B. Barc, M. Ryszka, J. Spurrell, M. Dampc, P. Limão-Vieira, R. Parajuli, N. J. Mason, and S. Eden, J. Chem. Phys. 139, 244311 (2013).

${ }^{42}$ R. Pandey, M. Ryszka, T. da Fonseca Cunha, M. Lalande, M. Dampc, P. Limão-Vieira, N. J. Mason, J. C. Poully, and S. Eden, Chem. Phys. Lett. 684, 233 (2017). 